\title{
Lift-off reduction and patella tracking in Total Knee Arthroplasty (TKA) using a 3D printed patient- specific knee model
}

Alexandra Mercader ( $\sim$ alexandra.mercader@tum.de)

Technical University of Munich, Garching by Munich

\section{Isabella Ostner}

Technical University of Munich, Garching by Munich

Freya Küppers

Technical University of Munich, Garching by Munich

Tim C. Lueth

Technical University of Munich, Garching by Munich

Heinz Röttinger

München Klinik

Amir H. Bigdeli

Surgical Clinic Munich South

\section{Research Article}

Keywords: Total Knee Arthroplasty, Patellofemoral Joint, 3D Printing, Patient-Specific, Kinematic Alignment, Measured Resection

Posted Date: November 30th, 2021

DOI: https://doi.org/10.21203/rs.3.rs-1064965/v1

License: (9) This work is licensed under a Creative Commons Attribution 4.0 International License. Read Full License 


\title{
Lift-off reduction and patella tracking in Total Knee Arthroplasty (TKA) using a 3D printed patient-specific knee model
}

\author{
Alexandra Mercader ${ }^{1, *,+}$, Isabella Ostner ${ }^{1}$, Freya Küppers ${ }^{1}$, Heinz Röttinger ${ }^{2,+}$, Amir H. \\ Bigdeli $^{3,+}$, and Tim C. Lueth ${ }^{1}$ \\ ${ }^{1}$ Technical University of Munich, Institute of Micro Technology and Medical Device Technology, Garching by Munich, \\ 85748, Germany \\ ${ }^{2}$ München Klinik, Department of Endoprosthetics, Munich, 81737, Germany \\ ${ }^{3}$ Surgical Clinic Munich South, The Department of Diagnostic and Interventional Radiology, Munich, 81379, \\ Germany \\ *alexandra.mercader@tum.de \\ +these authors contributed equally to this work
}

\begin{abstract}
The patellofemoral joint is greatly influenced by the geometry and position of the femur during knee flexion. After knee replacement surgery, the joint geometry is changed by the surface replacement and its kinematics by the orientation of the cutting planes planned by the surgeon. The technique presented in this study verifies the compatibility of the cutting method during total knee arthroplasty (TKA) with the geometry of the joint and the kinematics of knee flexion. To confirm the benefit of this method, three patient-specific knee models were printed in 3D. All models were made from the patient's CT data and the patient's measurement of flexion motion obtained with an infrared stereo camera. The models are operated according to three different techniques: Kinematic Alignment (KA), Measured Resection (MR) with 3 degrees of external rotation and MR with 9 degrees of external rotation. The resulting postoperative knees from the different alignment techniques are compared to the patient's preoperative knee. The results show that the movement of the patella is modified after surgery on the model. The different alignments also lead to a variation in the behavior of the tibiofemoral joint. Based on these three examples, MR with an external orientation of the prosthesis results in a patellar movement closer to the preoperative movement. The KA method also reproduces the patella movement almost identically, but a lift-off appears at the tibiofemoral joint. The error observed for each cutting method between the pre- and postoperative patella position varies by a maximum of 5 degrees of rotation and 5 $\mathrm{mm}$ of translation, showing that the configuration has an overall small impact on the patellar movement. This study of three cases shows the importance of the preparation of the operation and the adjustment of the prosthesis for each specific patient using the 3D printed model.
\end{abstract}

Keywords: Total Knee Arthroplasty, Patellofemoral Joint, 3D Printing, Patient-Specific, Kinematic Alignment, Measured Resection

\section{Introduction}

TKA is a total surface replacement surgery removing arthritic tissues of the femur and tibia in order to improve knee mobility. However, this operation is very controversial, as it only provides $80 \%$ satisfaction $^{1}$ among the patients. This implies that patients suffering from pain after the operation have to undergo a second operation, involving additional costs for the patient and the health care facilities. In the United States, about $20 \%$ of revision TKA procedures are performed due to mechanical loosening ${ }^{2}$. The main reason for this loosening is a malalignment of the prosthesis, resulting in a change in the kinematics of the tibiofemoral and patellofemoral joint ${ }^{3,4}$. Gasparini et al. ${ }^{5}$ have already pointed out the problem of patellar alignment measuring the forces involved in knee flexion. The Q-angle, or the angle between the line of the patellar ligament and the quadriceps, may be altered following surgery and lead to anterior postoperative pain. In a study involving 62 patients ${ }^{6}$, postoperative pain has been shown to result from an increased Q-angle and internal rotation of the femoral component of the prosthesis. The patellofemoral joint is modified as the patella follows the geometry of the femur surface (trochlear groove) which is being reconstructed in a different way after the operation, due to the prosthesis position and shape ${ }^{7}$. Internal rotation of the femoral component also leads to instability of the tibiofemoral joint. The joint instability is caused by a lift-off at the mid-flexion. This lift-off is not the direct cause of the pain, but this instability influences the lateral ligaments forces and therefore the kinematics 
of the knee ${ }^{8}$.

To understand which position of the prosthesis is best suited to avoid instability and reproduce preoperative motion, other types of experiments were performed on models. The models are either real or virtual. Cutting experiments comparing different surgery techniques were performed in the literature on cadaveric knees to gain a better understanding of the knee joint and the consequences of $\mathrm{TKA}^{4,9}$. The results of these experiments are controversial, showing however a preference for the kinematic alignment method ${ }^{10,11}$. This cutting method consists of restoring the preoperative alignment of the knee. Other methods such as mechanical alignment that aligns the hip, knee and ankle joints, gap-balancing that checks the tension in the lateral ligaments, or measured resection that restores the preoperative surface of the knee, are common surgical procedures for TKA. However, there is no single method that results in general patient satisfaction. Miozzari et al. ${ }^{12}$ compare gap balancing and measured resection showing that these two methods lead to the same outcomes. In a study by Nakajima et al. ${ }^{13}$, patient-reported outcomes have been in favor of kinematically aligned implants against measured resection technique. However, a review by Roussot et al. ${ }^{14}$ comparing mechanical alignment and kinematic alignment shows that despite the general preference for natural restoration of the knee axes with the kinematic alignment, a correlation between pain and prosthesis alignment has not been clearly established.

The complexity of the knee joint and the significant differences in geometry and motion between individual patients make it difficult to choose the best surgical technique. Computer models also provide insight into the influence of the prosthesis and the forces associated with the flexion motion. For the patellofemoral joint, Tzanetis et al. ${ }^{15}$ show that little difference is seen in the patella kinematics when changing the thickness of the insert. The model of Heegard et al. ${ }^{16}$ indicates that patellar tracking is closer to natural kinematics when the prosthesis is externally rotated. Watanabe et al. ${ }^{17}$ show that a lift-off and a tibio-femoral instability appears with varus alignment of the prosthesis. Kebbach et al. ${ }^{18}$ proved that the position and thickness of the patella implant influence contact forces and kinematics. These studies imply that a change in the surface of the femur influences the forces and the kinematics of the patellofemoral joint. However, no type of generalized alignment for all patients has been proven to reduce pain in primary knee replacement patients.

The observations in the literature show that postoperative pain is mainly due to prosthesis malalignment. However, these experiments are performed on cadaveric knees or computerized models, which does not allow the findings to be extended to current patients with pain. There is a need to compare the influence of the prosthesis position on the instability of the tibiofemoral joint and on the patellofemoral joint kinematics. For this purpose, this study presents the results of lift-off and patellar tracking for different prosthesis positions on specific 3D models of patient's knees. Kinematic alignment (KA) and measured resection (MR) with 3 and 9 degrees of external rotation are compared with the preoperative knee joint. These experiments are performed on three patients, two of them suffer from osteoporosis and one has a healthy knee.

\section{Materials and Methods}

\section{Planar representation of the patient-specific knee model}

Specific models of three patients were constructed similarly to the models used for the rollback measurement experiment in a previous publication ${ }^{19}$. In this new experiment, the movement of the tibia in relation to the femur of the patient is also recorded using customized hard shells to support the optical trackers. The position of each tracker is measured with a Vicra infrared stereo camera (Northern Digital, Inc., Waterloo, Ontario, Canada). The measured flexion is used to visualise the animated 3D model of the patient's knee made with CT data ${ }^{20}$. A four-bar mechanism is constructed with the measurement to reproduce the specific movement of the patient. The mathematics for the design of the mechanism are explained more thoroughly in the corresponding technical paper ${ }^{21}$. All computations and simulations are made with MATLAB.

Modeling the flexion movement by a four-bar mechanism constrains the tibia and femur in the same plane. This plane is calculated statistically from the patient's measurement data. The contact area between the femur and the tibia can then be represented in all planes parallel to this calculated plane. Knee flexion consists of two phases, first mostly a rotation of the femur on the tibia until about 20 degrees of flexion, then mostly a sliding motion, also called rollback. This explanation of knee flexion movement was described by Fischer ${ }^{22}$, who modeled the knee from the sagittal view. His study was performed in 2D and limited by the planar views of the scans. Contrary to Fischer, the knee models used in this study are computerized 3D surface models. The plane for the lateral view is computed statistically using the patient's flexion measurement, considering the six degrees of freedom of the knee movement. The selected plane is therefore patient-specific and different from the sagittal plane. The two phases of rolling and sliding motion are also visible on the 3D printed model. A simplified 2D visualization explains how the 3D model behaves. For this purpose, the contour of the femur and the tibia are modeled by a set of points $x_{\text {fem }}$ and $x_{t i b} \cdot x_{f e m}$ is a fixed contour, as only the tibia moves around the femur. The length $l_{1}, l_{2}$ and $l_{3}$ and angles $w_{1}, w_{2}$ and $w_{3}$ are represented in Figure 1, as well as the rotation points $A_{0}, B_{0}, A_{1}$ and $B_{1}$. The length and position of the four-bar linkage are computed with the algorithm described in a previous study ${ }^{21}$. The position of the four bar linkage is modeled by the following 


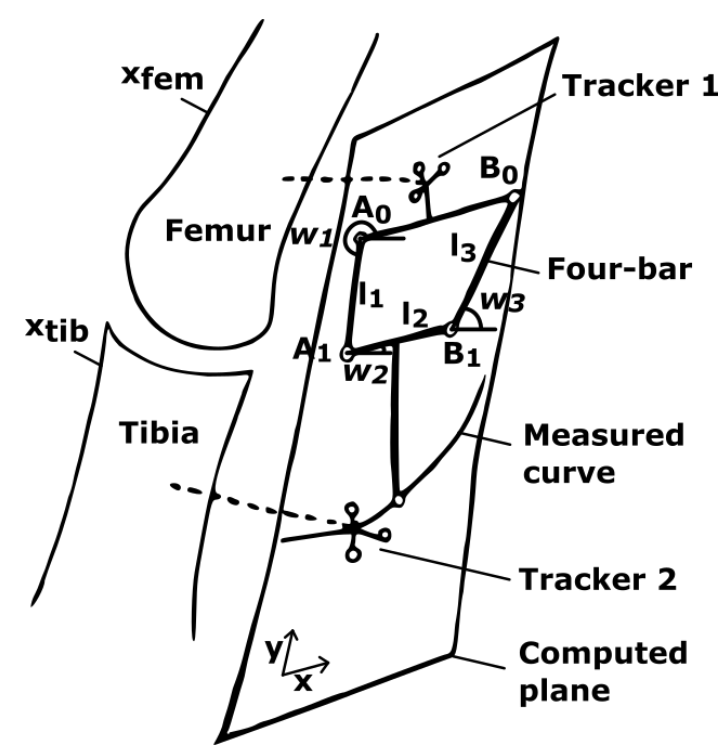

Figure 1. Illustration of the statistically computed plane from the 3D patient measurement and the four-bar mechanism reproducing the measured trajectory. The mechanism is physically connected to the femur and tibia bones.

system of equations:

$$
\left\{\begin{array}{l}
A_{0 x}+l_{1} \cos \left(w_{1}\right)+l_{2} \cos \left(w_{2}\right)+l_{3} \cos \left(w_{3}\right)=B_{0 x} \\
A_{0 y}+l_{1} \sin \left(w_{1}\right)+l_{2} \sin \left(w_{2}\right)+l_{3} \sin \left(w_{3}\right)=B_{0 y}
\end{array}\right.
$$

These equations represent the constraints on the two-dimensional coordinates of the mechanism that close the four-bar linkage loop. $w_{2}$ and $w_{3}$ have to be determined knowing all the other parameters. $w_{1}$ has simple values going from $168^{\circ}$ at the start position of the extended leg to $204^{\circ}$ at the maximum flexion with the mechanism. For this patient, $l_{1}=102 \mathrm{~mm}, l_{2}=100 \mathrm{~mm}$ and $l_{3}=61 \mathrm{~mm}$. The other parameters values are the following: $A_{0 x}=46 \mathrm{~mm}, A_{0 y}=140 \mathrm{~mm}, B_{0 x}=-4 \mathrm{~mm}$ and $B_{0 y}=93 \mathrm{~mm}$. Each patient has a specific knee movement, and therefore a specific four-bar mechanism that simulates the flexion for the model.

The contour of the femur and tibia are shown for each of the condyles. Two planes parallel to the four-bar linkage and passing through the center of the condyles are selected to visualize the rolling and sliding movement of the knee joint. The femur contour is fixed, while the tibia contour is animated simultaneously with the mechanism's coupler link. For this purpose, the homogeneous transformation matrix ${ }^{w} T_{2}$ of the coupler link of length $l_{2}$ in the world coordinates of the plane is defined as:

$$
{ }^{w} T_{2}=\left(\begin{array}{ccc}
\cos \left(w_{2}\right) & -\sin \left(w_{2}\right) & A_{1 x} \\
\sin \left(w_{2}\right) & \cos \left(w_{2}\right) & A_{1 y} \\
0 & 0 & 1
\end{array}\right)
$$

knowing that:

$$
\left\{\begin{array}{l}
A_{1 x}=A_{0 x}+l_{2} * \cos \left(w_{1}\right) \\
A_{1 y}=A_{0 y}+l_{2} * \sin \left(w_{1}\right)
\end{array}\right.
$$

The contour is then transformed into the coordinate system ${ }^{w} T_{2}(1)$ of the position 1 . The contour follows the coupler for the positions $i$ from 2 to 8 with the following formula:

$$
{ }^{w} x_{t i b}(i)=\left({ }^{w} T_{2}(i)^{w} T_{2}(1)^{-1}\right)^{w} x_{t i b}(1)
$$

In Figure 2, the flexion movement is illustrated for the lateral and medial condyle. The corresponding values for $w_{1}, w_{2}$ and the flexion angles of the knee for positions 1 to 8 are listed in Table 1 . The contour area of the medial condyle is significantly smaller than the one of the lateral condyle for this patient. In both cases, there is a predominant rotational movement up to about 40 degrees (position 4), and then a sliding movement for positions 4 to 8 , as described in the literature. The transition is remarkable because positions 4 to 8 intersect at a single point, visible as being darker, representing the narrower posterior surface of the femur. Sliding is therefore predominant. Thanks to this planar representation, the optimal cutting planes for the 


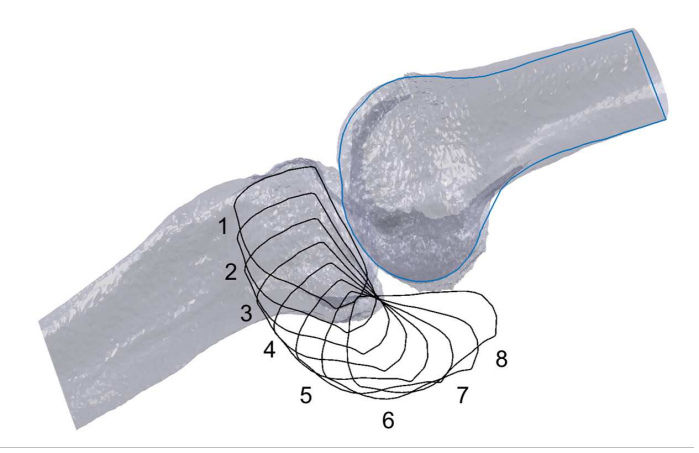

(a) Lateral condyle

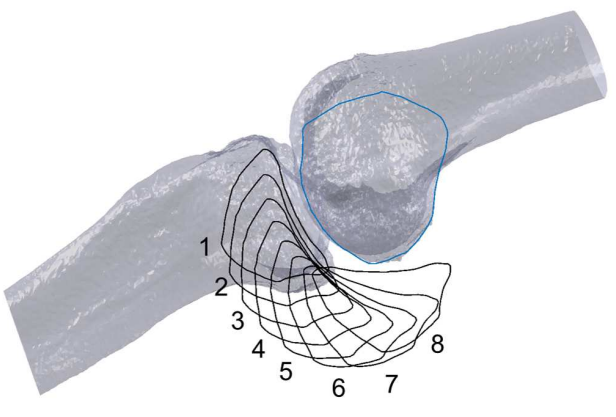

(b) Medial condyle

Figure 2. Representation of the femur and tibia contours for the lateral condyle (a) and the medial condyle (b) of a left knee of a patient, together with the transparent 3D surface model in the extended position for visualization. The flexion has been modeled through the four-bar linkage mechanism.

Table 1. Values of $w_{1}, w_{3}$ and the corresponding flexion angle of the tibia for the eight flexion steps illustrated in Figure 2

\begin{tabular}{||c|cccccccc||}
\hline Pose & 1 & 2 & 3 & 4 & 5 & 6 & 7 & 8 \\
\hline \hline$w_{1}(\mathrm{deg})$ & 168 & 173 & 179 & 184 & 189 & 194 & 199 & 204 \\
\hline$w_{3}(\mathrm{deg})$ & 32 & 43 & 54 & 67 & 81 & 98 & 117 & 142 \\
\hline Flexion $(\mathrm{deg})$ & 5 & 16 & 27 & 40 & 54 & 70 & 90 & 114 \\
\hline
\end{tabular}

placement of the prosthesis can be predicted. Indeed, the cutting planes can be defined perpendicular to the viewing plane. This results in an angle relative to the joint line with the positions 1 of the medial and lateral condyles and a second angle of rotation for the femoral cut relative to the posterior condylar axis with the positions 8 . This method makes it possible to verify the compatibility of the cut with the native kinematics of the knee. Based on positions 8 , if the goal is to maintain flexion in this plane, the hypothesis is to rotate the prosthesis externally with respect to the posterior condylar axis of the femur to reduce the lift-off and therefore maintain knee stability.

\section{D design of the patient-specific model with patella and measurements}

Once the mechanism has been designed by computer using Matlab and SG-Library ${ }^{23}$, the elements are 3D printed with Polylactic Acid (PLA) using the Fused Deposition Modeling (FDM) printing method. Patient's bones are being duplicated for the surgeon to perform each operation separately. For this, a silicone mold of the patient's knee is created and the mold is filled with polyurethane resin. The molding process is performed several times and the patient's knee is duplicated as many times as the number of operations to be performed on the model. Figure 3 illustrates a duplicated model of a femur and a tibia. At the filling location, the inner porous and red structure can be seen to reproduce the internal structure of the knee bones using Bioresin and red colors (Silitech $\mathrm{GmbH}$ ). The outside layer is a thin smooth layer of resin (Biresin G27) defining the exact shape of the patient's bones.

The patella is fixed to the tibial tuberosity with a 2 centimeter wide non-elastic band to imitate the patellar tendon. The length of the patellar tendon is measured on the patient's CT model. An elastic band of the same width is fixed to the top of the mechanism as the quadriceps muscle. The patient's model and the illustration of the patellar tendon as well as the quadriceps muscle are shown in Figure 4. The total length of the patellar tendon is measured manually on the computer using CT data as the distance between the lower part of the patella and the tibial tuberosity. For this patient, the patellar tendon has a length of 65 $\mathrm{mm}$. The patella can be fixed on the model bones with screws, being therefore interchangeable on different versions of the post-operative patient's bones.

An optical tracker is placed on the patella to measure its movement with an infrared stereo camera. Figure 5 shows the model in frontal and sagittal planes. The six degrees of freedom are calculated from the homogeneous transformation matrix of the optical tracker of the patella. Several flexions and extensions of the model are performed for the measurement. Figure 6 illustrates the three rotation's and three translation's measurements of the patella for each configuration. ${ }^{w} T_{p}$ is the transformation matrix of the patellar tracker in the world coordinates (the stereo camera). The patellar transformation matrix 


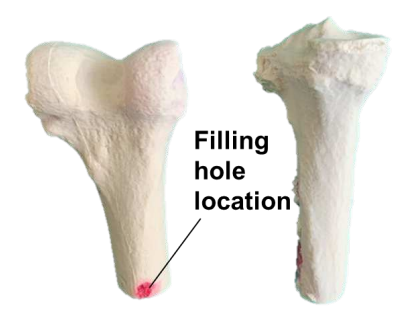

Figure 3. Illustration of a duplicate model of femur and tibia in polyurethane. The external structure is smooth and the internal structure has been foamed and colored to realize a porous structure of pink color.

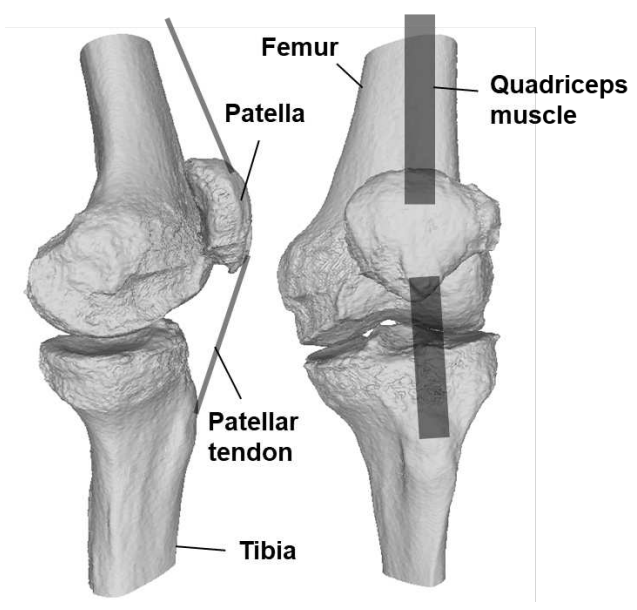

Figure 4. Illustration of the 3D model of the patient's knee with femur, tibia, patella, patellar tendon and quadriceps muscle. The length of the non-elastic patellar tendon is measured on the computer model. For this patient, the patellar tendon has a length of $65 \mathrm{~mm}$.
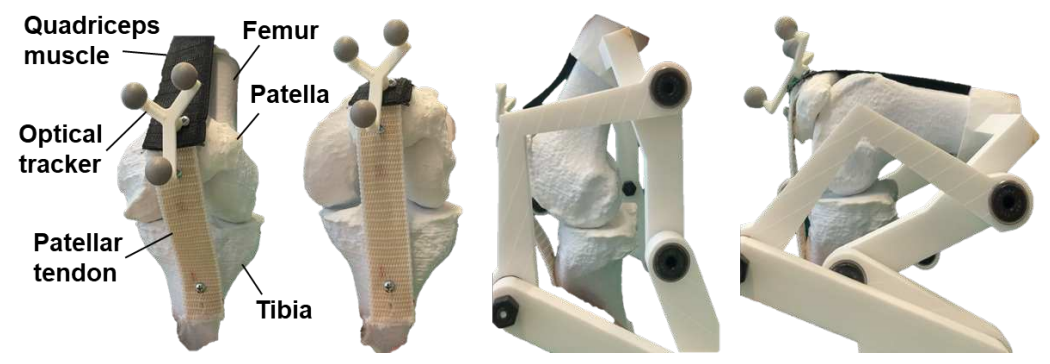

Figure 5. Picture of the 3D model of the patient's knee printed in the extension and flexion positions, in the frontal and sagittal planes. The four-bar mechanism constrains the knee flexion as measured on the patient. An optical tracker is attached to the patella for the experiments. 


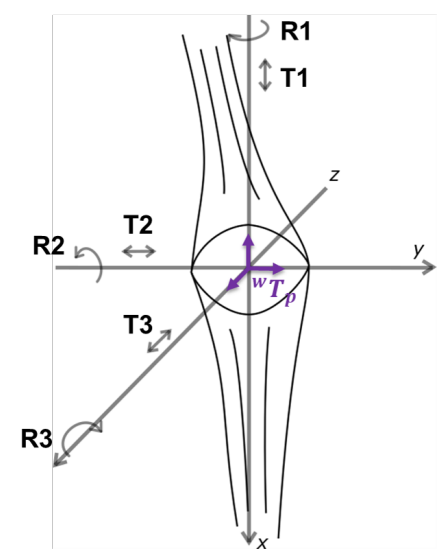

Figure 6. Illustration of the patella with its six degrees of freedom used in the results.

${ }^{e x t} T_{p}$ in the coordinate system of the patella in the extended position of the knee is as follows:

$$
{ }^{e x t} T_{p}=\left({ }^{w} T_{e x t}\right)^{-1 w} T_{p}
$$

The six degrees of freedom are computed as follows, considering the coefficients of the homogeneous transformation matrix of the patella as $t_{i j}$ where $i$ and $j$ are respectively the row and column index.

$$
\left\{\begin{array}{l}
R 2=\tan ^{-1} \frac{-t_{31}}{\sqrt{t_{11}^{2}+t_{21}^{2}}} \\
R 1=\tan ^{-1} \frac{t_{32} / \cos (R 2)}{t_{33} / \cos (R 2)} \\
R 3=\tan ^{-1} \frac{t_{21} / \cos (R 2)}{t_{11} / \cos (R 2)} \\
T 1=t_{14} \\
T 2=t_{24} \\
T 3=t_{34}
\end{array}\right.
$$

$R 1, R 2$ and $R 3$ correspond to the internal-external, the extension-flexion and the varius-valgus rotations respectively. $T 1$, $T 2, T 3$ are the medial-lateral, the proximal-distal and the anterior-posterior translations. Each of these degrees of freedom are compared for the three models in the following experiments.

\section{Operation on the model}

The operation on the model is made with standard instruments for TKA using the TC-PLUS PRIMARY set from Smith \& Nephew. The first technique being tested is measured resection following bony landmarks. For all experiments, the patella is naturally preserved, without resurfacing. Daines et al. ${ }^{24}$ explain how to define the cutting planes in the case of the measured resection technique. The distal cut is set at 6 degrees valgus relative to the intramedullary guide. The tibial cut is perpendicular to the longitudinal tibial axis. The rotation of 0 degree of the prosthesis is defined by the posterior condylar axis (PCA). MR of 3 and 9 degrees of external rotation are executed on each model. A kinematic alignment is also performed, following the distal femoral joint line for the distal cut and the PCA for the anterior and posterior cuts. The duplicated bones made with polyurethane resin that has been foamed up for bone imitation are being cutted as it would have been done on the patient. After positioning the prosthesis on the model, the resin bones are placed back into the four-bar linkage. The preoperative kinematics are conserved throught the mechanism so that lift-off and patellar tracking can be compared between the preoperative model and postoperative configurations as in Berger et al. ${ }^{25}$ to avoid patellofemoral complications. Figure 7 shows the model with duplicated bones and prosthesis after MR technique with 3 degrees of rotation. This model has an optical tracker placed on the patella to measure and compare patellar kinematics.

\section{Results}

A total of three knee models are made from the knees of three different patients. One patient has a healthy knee (patient 1 ) and the two others have osteoporosis (patient 2 and 3). For these three patients, the operation is performed and three prosthesis positions are tested and compared with the native knee model. 


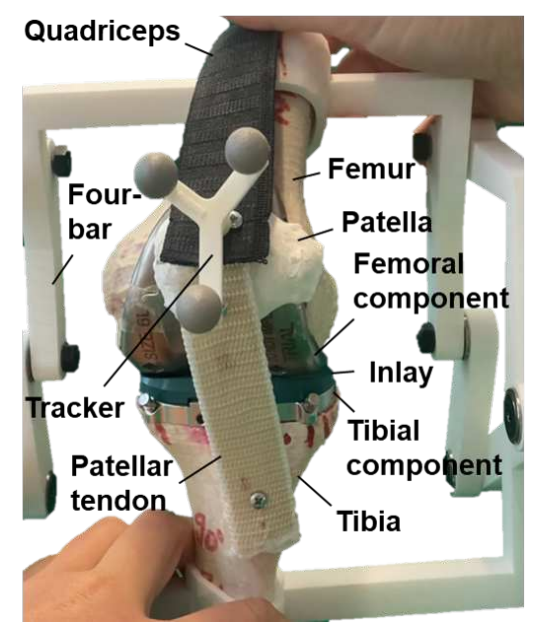

Figure 7. Picture of the 3D model of patient 1 with duplicated bones after prosthesis implantation using MR and 3 degrees of external rotation.

Table 2. Pre- and post-operative lateral lift-off values for the three models of the patients

\begin{tabular}{||c|cccc||}
\hline Configuration & MR 3 $^{\circ}$ & MR 9 $^{\circ}$ & KA & Natural \\
\hline \hline Lateral lift-off patient 1 $(\mathrm{mm})$ & 5 & $\mathbf{0}$ & 2 & 0 \\
\hline Lateral lift-off patient 2 $(\mathrm{mm})$ & 6 & $\mathbf{0}$ & 4 & 3 \\
\hline Lateral lift-off patient 3 $(\mathrm{mm})$ & 3 & 1 & 3 & 3 \\
\hline
\end{tabular}

\section{Lift-off reduction}

The lift-off is measured for each configuration and compared to the preoperative value. This parameter shows the influence of the kinematic constraints of the cruciate ligaments on the knee joint stability for each implant position. The results are listed in Table 2. For all three patients, the lift-off observed on the model is only lateral. The medial condyle does not present any lift-off. The measured values show an absence of lift-off for MR $9^{\circ}$ configuration for patients 1 and 2 , and $1 \mathrm{~mm}$ of lift-off for patient 3 , which favours a higher external rotation of the femoral component. The knees of patients 2 and 3 with osteoporosis had a lateral lift-off before the operation of $3 \mathrm{~mm}$. The kinematic alignment (KA) is the closest to this value for these patients, resulting in a postoperative lateral lift-off of 4 and $3 \mathrm{~mm}$, respectively. The largest lift-off is measured for the MR $3^{\circ}$ configuration in the case of patient 2. This case is illustrated in Figure 8. The experiment validates the hypothesis theoretically postulated from the two-dimensional view shown in Figure 2 that external rotation aligns the femoral surface with the flexion plane. This external rotation counterbalances the near symmetry of the prosthesis modifying the femur surface and thus the flexion movement. By constraining this flexion movement with the printed mechanism on the knee model, the best position of the prosthesis can be established from these experiments.

\section{Patellar kinematics}

Patellar motion is compared to preoperative motion for all three patients. On each model, the three operations techniques are being tested. The six degrees of freedom are measured with the tracker and the infrared stereo camera, for each patient and each prosthesis position. The detailed results for patient 2 are shown in figure 9 . For each degree of freedom, the measurement of three flexions of the model are interpolated by a polynomial of degree 6 according to the method of minimizing the squared distance between the model and the measurement. The curves show that an initial rotation occurs in the case of MR $3^{\circ}$, which illustrates a misplacement of the patella on the femoral component surface for the first ten degrees of flexion. This abrupt rotation can cause pain in the patient and is indicative of a lateral malalignment of the patella. For the following description of the patellar motion, the rotations and translations as described in Bull et al. ${ }^{26}$ are taken as reference. The results of patient 2 show a tendency for a lateral tilt of the patella, a flexion along the surface of the femur, a lateral rotation and a proximal, lateral and posterior translation along its own axes from the extension to the flexion position. More flexion and posterior translation of the patella for MR $9^{\circ}$ are observed for this patient. These both parameters illustrate a better rollback towards the back of the knee joint, meaning more stability and a higher angle range of the knee flexion. The greatest distance between the curves is 


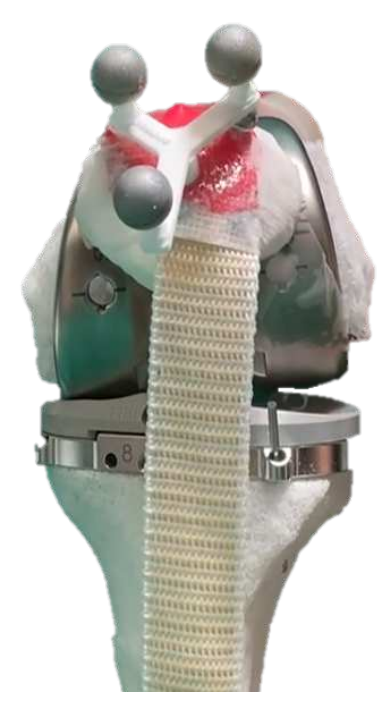

Figure 8. Illustration of the largest lateral lift-off for patient 2 with a configuration of MR $3^{\circ}$ at $90^{\circ}$ of flexion.

Table 3. Angle and translation error between the post-operative and the native knee curves for the three patients

\begin{tabular}{||c|ccc|ccc|ccc||}
\hline Configuration & \multicolumn{3}{|c|}{ MR 3 $^{\circ}$} & \multicolumn{3}{c|}{ MR 9 } & \multicolumn{3}{c||}{ KA } \\
\hline Patient number & 1 & 2 & 3 & 1 & 2 & 3 & 1 & 2 & 3 \\
\hline \hline Error R1 $\left(^{\circ}\right)$ & $\mathbf{2 . 7}$ & 3.9 & 6.0 & 5.0 & $\mathbf{3 . 2}$ & $\mathbf{3 . 6}$ & 4.2 & 4.7 & 5.0 \\
\hline Error R2 $\left(^{\circ}\right)$ & 3.5 & 4.2 & 3.1 & 1.9 & 11.7 & 1.1 & $\mathbf{1 . 7}$ & $\mathbf{1 . 9}$ & $\mathbf{0 . 8}$ \\
\hline Error R3 $\left(^{\circ}\right)$ & 2.0 & 2.7 & 1.0 & $\mathbf{1 . 8}$ & $\mathbf{1 . 6}$ & 1.5 & 2.6 & 1.8 & $\mathbf{0 . 7}$ \\
\hline Error T1 $(\mathrm{mm})$ & 8.1 & $\mathbf{1 . 0}$ & 0.7 & $\mathbf{3 . 3}$ & 2.1 & 0.7 & 5.3 & 1.9 & $\mathbf{0 . 6}$ \\
\hline Error T2 $(\mathrm{mm})$ & 5.5 & 6.8 & 1.9 & $\mathbf{3 . 3}$ & $\mathbf{2 . 6}$ & 2.5 & 3.6 & 4.4 & $\mathbf{0 . 9}$ \\
\hline Error T3 $(\mathrm{mm})$ & 9.7 & $\mathbf{2 . 1}$ & 5.4 & $\mathbf{2 . 4}$ & 11.2 & $\mathbf{1 . 8}$ & 4.8 & 4.3 & 4.4 \\
\hline
\end{tabular}

observed for lateral translation. KA leads to a very little medial-lateral translation, while MR $9^{\circ}$ results in a lateral translation similar to the native knee, which stabilizes after 40 degrees of flexion. The patella of the native knee goes back to its initial position after medial translation at about 40 degrees of flexion. For MR $3^{\circ}$, the medial translation is greater than all other configurations at 30 degrees, showing a higher change in the direction of patellar tendon tension forces and thus possible pain for the patient.

Table 3 lists the rotation and translation error between the postoperative curve and the natural knee curve. This error is calculated for each of the six degrees of freedom, for each of the three models. Values in bold correspond to the minimum error for a patient for a given degree of freedom. In total, MR $9^{\circ}$ with 9 minima has the most minimum values, compared to MR $3^{\circ}$ having only three minimum values. For patients 1 and 2 , the MR $9^{\circ}$ configuration seems to provide a patellar motion with the least error to the natural motion. For patient 3, on the contrary, the KA configuration has the most minimum error values. However, when comparing KA values with MR $9^{\circ}$, the sum of the rotational error is $6.2^{\circ}$ for $\mathrm{MR} 9^{\circ}$ and $6.5^{\circ}$ for KA, and the sum of the translational error is $5 \mathrm{~mm}$ for MR $9^{\circ}$ and $5.9 \mathrm{~mm}$ for KA. Thus, there are fewer extreme values for MR $9^{\circ}$ for patient 3 , which again still shows a preference for an external rotation of the prosthesis.

\section{Discussion}

In this research, lift-off and patellar tracking are measured using a custom 3D printed knee model. This model allows to observe the influence of the kinematic constraints of the native knee ligaments on the positioning of the prosthesis. During the experiments, the hypothesis made from the 2D visualization of the patient's movement was confirmed on the three models. The plane in which the movement is approximated shows a difference in size and in position in the plane between the medial and lateral condyles of the femur. This implies that an external rotation of the femur prosthesis would restore the balance and allow a flexion located in the computed plane. In the experiments, the lift-off is reduced and the patellar motion is closest to the native motion for all three patients for an external orientation of the femoral component. 

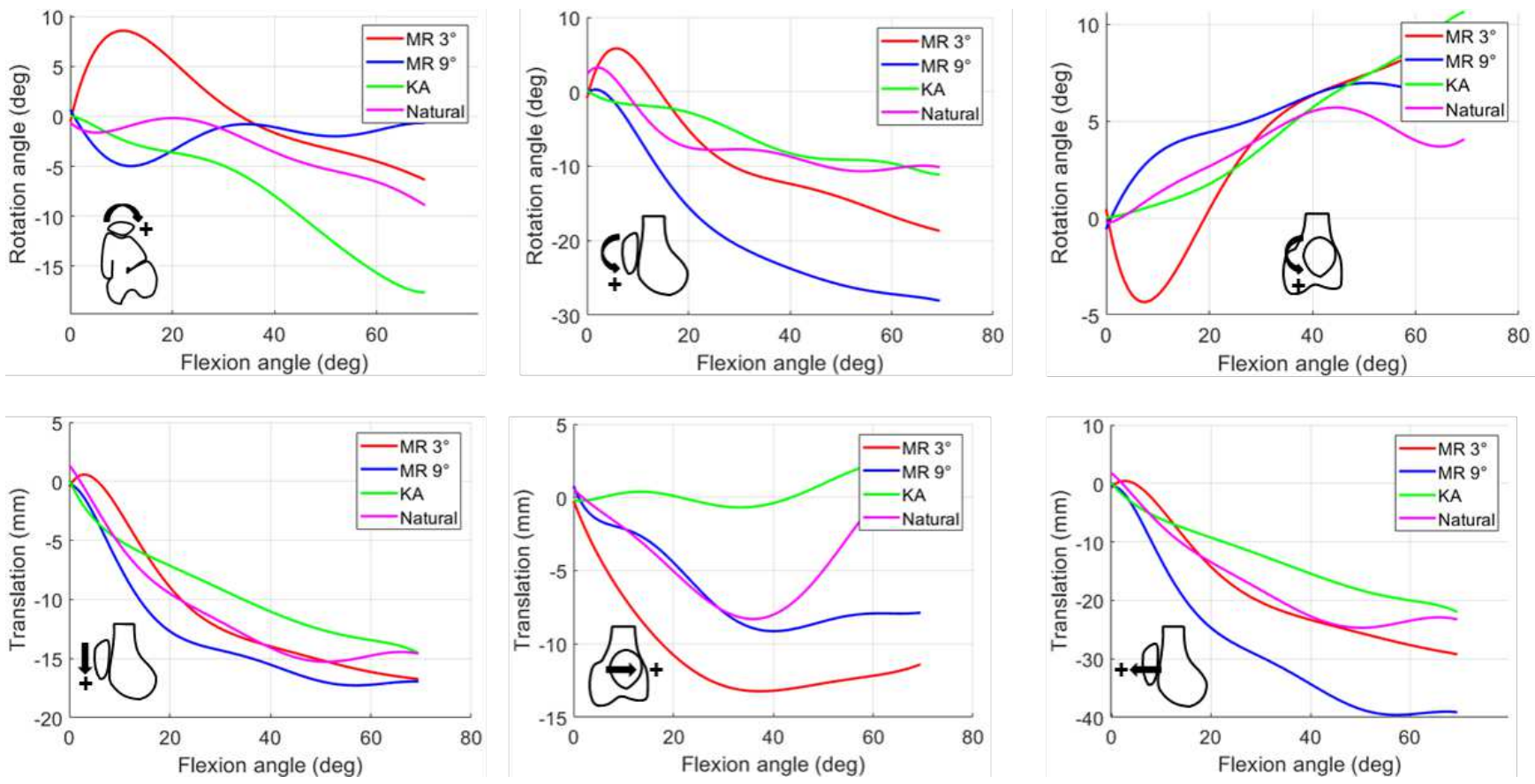

Figure 9. Pre- and postoperative patellar kinematics of patient 2 showing the differences between MR $3^{\circ}, \mathrm{MR}^{\circ}$ and KA configurations, for each degree of freedom.

A study of 25 patients by Insall et al. ${ }^{27}$ showed a correlation between the lift-off and the alignment of the femoral component. For each patient with lift-off, malalignment of the femoral component was measured on CT images. The lift-off was maximally $2.3 \mathrm{~mm}$ and mainly lateral. In a study by MacDessi et al. ${ }^{28}$, the mechanical alignment was shown to cause a greater lift-off than the kinematic alignment. The same observation was made by Blakeney ${ }^{29}$, demonstrating that mechanical alignment along the transepicondylar axis (TEA) or the posterior condylar axis (PCA) causes more instability during flexion. In this study, 51\% of the knees according to TEA and 37\% according to PCA had a lift-off of more than $3 \mathrm{~mm}$. The lift-off was more medial for varus knees and lateral for valgus knees. Lateral lift-off was also observed more often than medial lift-off in a study by Key et al. ${ }^{30}$ using fluoroscopy on patients after total knee arthroplasty while performing activity. More specifically, two studies by Scuder $^{31}$ and Heyse ${ }^{32}$, show a similar reduction in lift-off when the femoral component is externally rotated. However, despite the general improvement in stability with improved prosthesis positioning, studies show that there is no direct correlation between femoral component rotation and postoperative patient satisfaction, as observed by Corona et al. ${ }^{33}$.

The insatisfaction comes from pain and patients suffer mainly from anterior knee pain. This pain is primarily caused by the patellar tendon, triggered by a lateral displacement of the patella or a torsion ${ }^{34,35}$. In the literature, clinical studies show that an internal rotation of the femoral component causes more pain than an external rotation, especially for the patients involved in the research of Barrack et al. ${ }^{36}$. In general, malalignment of the femoral implant is an important cause of pain due to a change in the geometry and thus in the surface of the anterior femoral surface and of the femoral condyles. In a study on cadaveric knees by Donell ${ }^{37}$ and on patients by $\mathrm{Oh} \mathrm{Ko}^{10}$, on the contrary, no relationship between rotation of the femoral component and patellar tracking was observed. Computer simulations, as presented by Heegard et al. ${ }^{16}$ and Xie et al. ${ }^{38}$ prove that external rotation has less impact on patellar tracking than internal rotation. Regardless of the findings, there is evidence that femoral component rotation has an impact on patellofemoral joint kinematics, as demonstrated by Anglin et al. ${ }^{39}$ on cadaveric knee specimens. The focus of the research in the literature, however, is on the lateral movement of the patella and on the patellar tilt and not on the three-dimensional movement.

Although the literature results are controversial, they all show that there is a relationship between the prosthesis and patellar tracking, influencing patient satisfaction. The position of the femoral component also influences the lift-off and more generally the surgery outcomes. By combining these statements with the results of this paper, lift-off leads to knee instability, patellar tracking is influenced by component rotation and influences patient satisfaction. 


\section{Conclusion}

In this paper, the hypothesis that external rotation of the femoral component reduces the lift-off and improves patellar tracking was confirmed by experiments performed on three specific knee models. These results coincide with many studies presented in the literature. The conducted measurements provide insight into the tibiofemoral joint and the three-dimensional movement of the patella, comparing different configurations of the femoral component with the native knee. By simulating the movement of the patient's knee as closely as possible to the reality, the impact of the position of the prosthesis on the kinematics could be understood in specific cases of osteoarthritis but also in healthy knees. The results show a reduced lift-off in the case of MR $9^{\circ}$, but a similar between the native knee and KA. For patellar tracking, MR $9^{\circ}$ is more suitable for the arthritic knees, and KA for the healthy knees. However, this study also shows that each patient is unique, and a comprehensive analysis of the patient's kinematics is essential to determine the optimal position of the prosthesis in order to achieve a compromise between the tibiofemoral joint and the patellofemoral joint.

\section{References}

1. Kahlenberg, C. A. et al. Patient satisfaction after total knee replacement: a systematic review. HSS J. 14, 192-201 (2018).

2. Delanois, R. E. et al. Current epidemiology of revision total knee arthroplasty in the united states. The J. arthroplasty 32, 2663-2668 (2017).

3. Mathis, D. T., Lohrer, L., Amsler, F. \& Hirschmann, M. T. Reasons for failure in primary total knee arthroplasty-an analysis of prospectively collected registry data. J. Orthop. 23, 60-66 (2021).

4. Faschingbauer, M. et al. The tibial cut influences the patellofemoral knee kinematics and pressure distribution in total knee arthroplasty with constitutional varus alignment. Knee Surgery, Sports Traumatol. Arthrosc. 28, 3258-3269 (2020).

5. Gasparini, G., Familiari, F. \& Ranuccio, F. Patellar malalignment treatment in total knee arthroplasty. Joints 1, 10 (2013).

6. Planckaert, C. et al. Total knee arthroplasty with unexplained pain: new insights from kinematics. Arch. orthopaedic trauma surgery 138, 553-561 (2018).

7. Hochreiter, B., Hirschmann, M. T., Amsler, F. \& Behrend, H. Highly variable tibial tubercle-trochlear groove distance (tt-tg) in osteoarthritic knees should be considered when performing tka. Knee Surgery, Sports Traumatol. Arthrosc. 27, 1403-1409 (2019).

8. Mathis, D. T. et al. Correlations of typical pain patterns with spect/ct findings in unhappy patients after total knee arthroplasty. Knee Surgery, Sports Traumatol. Arthrosc. 1-17 (2021).

9. Tanikawa, H., Tada, M., Harato, K., Okuma, K. \& Nagura, T. Influence of total knee arthroplasty on patellar kinematics and patellofemoral pressure. The J. arthroplasty 32, 280-285 (2017).

10. Koh, I. J. et al. Kinematically aligned total knee arthroplasty reproduces more native rollback and laxity than mechanically aligned total knee arthroplasty: a matched pair cadaveric study. Orthop. \& Traumatol. Surg. \& Res. 105, 605-611 (2019).

11. Keshmiri, A. et al. Kinematic alignment in total knee arthroplasty leads to a better restoration of patellar kinematics compared to mechanic alignment. Knee Surgery, Sports Traumatol. Arthrosc. 27, 1529-1534 (2019).

12. Miozzari, H. H., Armand, S., Turcot, K., Lübbeke, A. \& Bonnefoy-Mazure, A. Gait analysis 1 year after primary tka: no difference between gap balancing and measured resection technique. The journal knee surgery (2019).

13. Nakajima, A. et al. Association between limb alignment and patient-reported outcomes after total knee arthroplasty using an implant that reproduces anatomical geometry. J. orthopaedic surgery research 13, 1-8 (2018).

14. Roussot, M. A., Vles, G. F. \& Oussedik, S. Clinical outcomes of kinematic alignment versus mechanical alignment in total knee arthroplasty: a systematic review. EFORT Open Rev. 5, 486-497 (2020).

15. Tzanetis, P., Marra, M. A., Fluit, R., Koopman, B. \& Verdonschot, N. Biomechanical consequences of tibial insert thickness after total knee arthroplasty: A musculoskeletal simulation study. Appl. Sci. 11, 2423 (2021).

16. Heegaard, J., Leyvraz, P. \& Hovey, C. A computer model to simulate patellar biomechanics following total knee replacement: the effects of femoral component alignment. Clin. Biomech. 16, 415-423 (2001).

17. Watanabe, M. et al. Varus femoral and tibial coronal alignments result in different kinematics and kinetics after total knee arthroplasty. Knee Surgery, Sports Traumatol. Arthrosc. 25, 3459-3466 (2017).

18. Kebbach, M. et al. Musculoskeletal multibody simulation analysis on the impact of patellar component design and positioning on joint dynamics after unconstrained total knee arthroplasty. Materials 13, 2365 (2020). 
19. Mercader, A., Röttinger, T., Bigdeli, A., Lüth, T. C. \& Röttinger, H. A patient-specific $3 \mathrm{~d}$ model of the knee to compare the femoral rollback before and after total knee arthroplasty (tka). J. Exp. Orthop. 8, 1-10 (2021).

20. Mercader, A., Roettinger, H., Bigdeli, A. \& Lueth, T. C. Visualization of patient's knee movement and joint contact area during knee flexion for orthopaedic surgery planing validation. Comput. Methods Biomech. Biomed. Eng. Imaging \& Vis. $1-8$ (2020).

21. Mercader, A., Roettinger, H., Bigdeli, A. \& Lueth, T. C. A 3d printed mechanical model of the knee to detect and avoid total knee replacement surgery errors. In International Conference on Robotics and Automation (ICRA) (2021).

22. Fischer, O. Kinematik organischer Gelenke, vol. 18 (Vieweg, 1907).

23. Lueth, T. C. Sg-lib-matlab-toolbox (https://github.com/timlueth/sg-lib-matlab-toolbox). GitHub (2021).

24. Daines, B. K. \& Dennis, D. A. Gap balancing vs. measured resection technique in total knee arthroplasty. Clin. orthopedic surgery 6, 1-8 (2014).

25. Berger, R., Crossett, L., Jacobs, J. \& Rubash, H. Malrotation causing patellofemoral complications after total knee arthroplasty. Clin. Orthop. Relat. Res. 356, 144-153 (1998).

26. Bull, A., Katchburian, M., Shih, Y. \& Amis, A. Standardisation of the description of patellofemoral motion and comparison between different techniques. Knee Surgery, Sports Traumatol. Arthrosc. 10, 184-193 (2002).

27. Insall, J. N. et al. Correlation between condylar lift-off and femoral component alignment. Clin. Orthop. Relat. Res. 403, $143-152$ (2002).

28. MacDessi, S. J. et al. Restoring the constitutional alignment with a restrictive kinematic protocol improves quantitative soft-tissue balance in total knee arthroplasty: a randomized controlled trial. The bone \& joint journal 102, 117-124 (2020).

29. Blakeney, W., Beaulieu, Y., Kiss, M.-O., Rivière, C. \& Vendittoli, P.-A. Less gap imbalance with restricted kinematic alignment than with mechanically aligned total knee arthroplasty: simulations on 3-d bone models created from ct-scans. Acta orthopaedica 90, 602-609 (2019).

30. Key, S. et al. Does lateral lift-off occur in static and dynamic activity in a medially spherical total knee arthroplasty? a pulsed-fluoroscopic investigation. Bone \& joint research 8, 207-215 (2019).

31. Scuderi, G. R., Komistek, R. D., Dennis, D. A. \& Insall, J. N. The impact of femoral component rotational alignment on condylar lift-off. Clin. Orthop. Relat. Res. 410, 148-154 (2003).

32. Heyse, T. J. et al. Internal femoral component malrotation in tka significantly alters tibiofemoral kinematics. Knee Surgery, Sports Traumatol. Arthrosc. 26, 1767-1775 (2018).

33. Corona, K. et al. Femoral component malrotation is not correlated with poor clinical outcomes after total knee arthroplasty. Knee Surgery, Sports Traumatol. Arthrosc. 28, 3879-3887 (2020).

34. Putman, S., Boureau, F., Girard, J., Migaud, H. \& Pasquier, G. Patellar complications after total knee arthroplasty. Orthop. \& Traumatol. Surg. \& Res. 105, S43-S51 (2019).

35. Matz, J., Lanting, B. A. \& Howard, J. L. Understanding the patellofemoral joint in total knee arthroplasty. Can. J. Surg. 62, 57 (2019).

36. Barrack, R. L., Schrader, T., Bertot, A. J., Wolfe, M. W. \& Myers, L. Component rotation and anterior knee pain after total knee arthroplasty. Clin. Orthop. Relat. Res. (1976-2007) 392, 46-55 (2001).

37. Donell, S. Patellar tracking in primary total knee arthroplasty. EFORT open reviews 3, 106-113 (2018).

38. Xie, X. et al. Effect of rotational prosthetic alignment variation on tibiofemoral contact pressure distribution and joint kinematics in total knee replacement. Proc. Inst. Mech. Eng. Part H: J. Eng. Medicine 231, 1034-1047 (2017).

39. Anglin, C. et al. Determinants of patellar tracking in total knee arthroplasty. Clin. Biomech. 23, 900-910 (2008).

\section{Acknowledgements}

We would like to thank all the authors for their contributions and especially the clinicians involved in this project for their help in understanding the techniques and challenges of the operation.

\section{Author contributions statement}

A.M. and H.R. conceived and conducted the experiments and analysed the results. A.B. provided all anonymous patient data. I.O. and F.K. are both students of TUM that contributed to the programming of the mathematical method and to the 3D construction of the model. T.L. conducted the team as a supervisor. All authors reviewed the manuscript. 


\section{Additional information}

No potential competing interest. Informed consent was obtained from the patients for the measurement of the knee movement with the infrared camera. The CT scans were collected as part of routine diagnosis and treatment. 